London SES 8AZ, England).

COMMENT. In this study conducted only in boys, inattentive behavior is a developmental risk factor for impairment of general cognitive functioning, poor reading and language skills, and a low self-esteem in school. In contrast to boys with hyperactivity and combined $\mathrm{ADHD}$, pure inattentive behavior is not associated with increased susceptibility to conduct problems or disturbed family relationships. Early recognition of ADD-inattentive subtype should allow prompt interventional treatment. Although boys outnumber girls with a $4: 1$ ratio for ADHD-HI subtype, the sex ratio is lower (2:1) for the ADHD-Inattentive type. Inattentiveness is relatively more prevalent among girls with ADHD, and any sexrelated cognitive and social risk factors would be of interest.

Parent-rated psychosocial correlates in preschool ADHD children.

In a study of 25 children with ADHD (21 males, 4 females; mean age 4.8 years), compared to 25 normal controls, at the IWK-Grace Health Centre, Halifax, Canada, parents rated their ADHD children as significantiy more aggressive, more demanding, less socially skilled, and less compliant. In contrast, the children perceived themselves as equally competent and as socially accepted as their peers. The parents viewed themselves as less competent parents. (DeWolfe NA, Byrne JM, Bawden HN. ADHD in preschool children: parent-rated psychosocial correlates. Dev Med Child Neurol Dec 2000;42:825-830). Presumably, these children had ADHDcombined type. No distinction of subtypes was made.

\title{
MAGNETIC BRAIN STIMULATION IN ADHD
}

Transcranial magnetic brain stimulation was performed in 27 children and adolescents, aged 4 to 18 years, with ADHD in the Services of Pediatric Neurology and Clinical Neurophysiology, Miguel Servet Hospital, Zaragoza, Spain. The motor evoked potential was recorded at the biceps brachii. Central motor conduction time mean value, calculated by cervical root stimulation, was very significantly increased $(\mathrm{P}<0.001)$ compared to normal controls. The finding reflects a delay in the maturation of the corticomotor-neuronal system. A side-to-side difference in the central motor conduction times in the ADHD group ( $\mathrm{P}=.03)$ suggests an hemispheric asymmetry in maturation. (Ucles P, Serrano J-L, Rosa F. Central conduction time of magnetic brain stimulation in attention-deficit hyperactivity disorder. I Child Neurol Nov 2000;15:723-728). (Respond: Dr Paulino Ucles, Almagro, 11 7-B, E-50004 Zaragoza, Spain).

COMMENT. Central motor conduction time in ADHD measured by transcranial magnetic brain stimulation may provide assistance in diagnosis and evidence supporting the theory of delayed cerebral maturation.

\section{SEIZURE DISORDERS}

\section{BEHAVIOR PROBLEMS ANTEDATING EPILEPSY ONSET}

The prevalence and nature of behavior problems among 224 children (ages 4 to 14 years) with epilepsy, in the six month period before the first recognized seizure, were studied at the Indiana School of Nursing, Indianapolis. Compared to their 135 healthy siblings, children with seizures had a higher than expected rate of antecedent behavior problems, with $32 \%$ in the clinical or at-risk range. Those with previous events suggestive of seizures had a $39 \%$ risk of behavior disorders before the first recognized seizure occurred. Children with seizures, especially 
boys, had higher scores than their siblings in total behavior problems, and in internalizing, attention, thought, and somatic complaints. Children with partial seizures showed higher rates of behavior problems than those with primary generalized epilepsy syndromes, regardless of previous seizures. Behavior problems are an integral component of epilepsy syndromes, and may occur independently and preceding the onset of clinical seizures. (Austin JK, Harezlak J, Dunn DW et al. Behavior problems in children before first recognized seizures. Pediatrics January 2001;107:115-122). (Reprints: Joan K Austin DNS, RN, Indiana University School of Nursing, 1111 Middle Drive, NU403, Indianapolis, IN 46202).

COMMENT. Behavior problems sometimes precede the onset of epilepsy and occur independently of seizures and anticonvulsant drugs. Children who develop partial seizures are at greater risk of antecendent behavior disorders than those with primary generalized epilepsy syndromes. The increased rate of attentional problems evident in the children with epilepsy in the above study, and especially in those with previous symptoms suggestive of seizures, provides support for the theory of transient cognitive impairment (TCI) associated with subclinical seizures (Aicardi J. Epilepsy as a non-paroxysmal disorder. Acta Neuropediatr 1996;2:249-257; Gonzalez-Garrido AA et al. Clin Electroencephalogr Oct 2000;31:175180; Ped Neur Briefs October 2000;14:74-75). As reviewed in our October issue of Ped Neur Briefs, children with resistant complex partial seizures have more severe thought disorder and cognitive impairments than those with primary generalized epilepsy (Caplan R et al. Epilepsia Oct 2000;41: Suppl 7;88).

In a recent study of BECTS at the Medical College of Georgia, Augusta, GA, of 22 children with centrotemporal spikes without clinical seizures, $31 \%$ had behavior problems and $17 \%$ had learning disabilities. So-called benign rolandic epilepsy may not be benign in neurodevelopmental outcome. (Yung AWY et al. Pediatr Neurol Nov 2000;23:391-395).

Antiepileptic treatment of children with behavioral and attention disorders and epileptiform EEGs without clinical seizures is controversial and of questionable value (Ped Neur Briefs Oct 2000;14:74). On the positive side, an improvement in psychosocial functioning with AED treatment was correlated with reduction in subclinical EEG epileptiform discharges in 8 of 10 children (Marston D et al. Dev Med Child Neurol 1993;35:574-581). The opposite, negative results were reported following treatment of a group of 8 children with behavior disorders and EEG epileptiform discharges without clinical seizures; valproate had adverse effects on learning, memory, and behavior (Ronen GM et al. Dev Med Child Neurol 2000;42:751-755; Ped Neur Briefs Dec 2000;14:92). The need for controlled studies of the effects of various AEDs on TCI and behavior in children with epileptiform EEGs without seizures is indicated.

\section{SHUDDERING ATTACKS}

The pathophysiology of shuddering attacks in 4 infants, ages 8 to 14 months, was studied using video-electroencephalographic monitoring, at the Epilepsy Center, Nishi-Niigata Central National Hospital, Niigata, Japan. Attacks occurred in clusters while awake, from a maximum of 5 to $>100$ daily, and a duration of 5-6 seconds. They included staring, straining, and shivering involving the head and upper extremities, without impairment of consciousness. Unusual movements included rare opisthotonic postures, clenching of teeth and hands, and eye deviation. Changing of diapers and being fed at meal times would often precipitate attacks. At follow-up at 25 to 45 months of age, attacks had resolved in one and occurred rarely (weekly to once every 3 months) in 3 . Three 